\title{
Introduction to Quality Improvement and Systems-Based Practice: a Two-Part Module for Clinical Clerkship Students
}

\author{
Galina Gheihman, $M D^{7,2}$, Brent P. Forester, $M D, M S C^{3,4,5}$, Niraj Sharma, MD, MPH $H^{6,7}$, \\ Cynthia So-Armah, MD, MPH ${ }^{7}$, Kathleen A. Wittels, $M D^{8}$, and Tracey A. Milligan, $M D^{2}$
}

'Department of Medicine, Brigham and Women's Hospital, Boston, MA, USA; ²Department of Neurology, Brigham and Women's Hospital, Boston, MA, USA; ${ }^{3}$ Division of Geriatric Psychiatry, McLean Hospital, Belmont, MA, USA; ${ }^{4}$ Partners Population Health, Partners HealthCare, Boston, MA, USA; ${ }^{5}$ Department of Psychiatry, Harvard Medical School, Boston, MA, USA; 'Internal Medicine-Pediatrics Residency Program, Brigham and Women's Hospital/Boston Children's Hospital, Boston, MA, USA; ${ }^{7}$ Departments of Medicine and Pediatrics, Harvard Medical School, Boston, MA, USA:

${ }^{8}$ Department of Emergency Medicine, Brigham and Women's Hospital, Boston, MA, USA

$\mathrm{J}$ Gen Intern Med 35(12):3742-4

DOI: $10.1007 / \mathrm{s} 11606-020-05788-0$

c) Society of General Internal Medicine 2020

\section{INTRODUCTION}

Healthcare systems and medical schools acknowledge the importance of developing students' skills in quality improvement (QI), health systems thinking, and delivery science. The Accreditation Council for Graduate Medical Education requires training at the graduate level ${ }^{1}$ and undergraduate curricula are increasingly emphasizing these topics. While medical schools are introducing QI concepts in the classroom, few provide opportunities to apply them clinically. ${ }^{2}$

Clerkships offer an opportunity to build student-centered, clinically integrated QI knowledge and skills. We designed a two-session module introducing students to basic concepts in patient safety and QI. We report an evaluation of this pilot and next steps for curriculum development.

\section{METHODS}

A two-part module was developed from a literature review and the authors' prior experience. The module asked students to identify a systems failure, investigate contributing factors, and brainstorm interventions, bookended by two one-hour discussions one month apart facilitated by faculty with QI expertise. A detailed session guide and key concepts sheet were created to guide student preparation and assist facilitators. The pilot ran April-May 2019, delivered to 59 students at a large urban US medical school during the clerkship year.

Students completed pre- and post-module surveys to assess their knowledge and understanding of basic concepts in QI and patient safety. Students self-reported their level of understanding or confidence on a 10-point scale as well as their attitudes about QI and patient safety. We assessed the module's

Prior Presentations None.

Received November 13, 2019

Accepted March 9, 2020

Published online March 23, 2020 overall quality and students' satisfaction with content and format. Surveys were distributed via email and statistics were completed in Excel (Microsoft Office, Seattle, WA). We surveyed faculty on the session's value and ease of facilitation.

\section{RESULTS}

Forty-three students completed the pre-module survey (43/59; $73 \%)$ and 31 completed the post-module survey $(31 / 59 ; 53 \%)$.

We found a significant increase in students' self-rating of their level of understanding of basic concepts within patient safety and QI, basic steps for improving systems, and the role they could play as students (Table 1A). Similar significant improvements were seen in students' self-ratings of their skills (Table 1B). After the session, all students "Agreed"/"Strongly Agreed" that "It is important for medical students and physicians to be able to identify errors and contribute to a system of patient safety and QI.”

A majority of students $(25 / 31 ; 81 \%)$ "Agreed"/“Strongly Agreed" that "These two sessions have increased my interest in patient safety and QI." Likewise, 74\% (23/31) "Agreed"/ "Strongly Agreed" that these sessions should continue in the future. Ninety percent (28/31) "Agreed"/"Strongly Agreed" having a QI-trained facilitator contributed significantly to their learning.

\section{Suggestions for Improvement}

Qualitative analysis of open-responses identified effective elements of the sessions and suggestions for improvement (Table 2). Novel ideas included introducing students to ongoing QI initiatives at our institution, and feeding forward student cases and/or ideas for improvement to the hospital's formal QI and safety infrastructure.

\section{DISCUSSION}

Our module was well received by clinical clerkship students. We demonstrated two one-hour sessions can be feasibly 
Table 1 Students' self-rating of knowledge and skills prior to the module (Pre-Module) and following the module (Post-Module)

\begin{tabular}{|c|c|c|c|}
\hline$\overline{\text { Question domain and question }}$ & $\begin{array}{l}\text { Pre-module } \\
\text { Mean (St Dev) } \\
N=43\end{array}$ & $\begin{array}{l}\text { Post-module } \\
\text { Mean (St Dev) } \\
N=31\end{array}$ & $\begin{array}{l}\text { Significance } \\
(2 \text { sample } t \text { test, } \\
\text { alpha }=0.05)\end{array}$ \\
\hline
\end{tabular}

A. Knowledge

Question: Please rate your level of understanding of the following concepts

(1 low; 10 high)

Basic concepts within patient safety (e.g., adverse events, near misses, culture of safety) - $6.05(2.01)$

The basic steps for improving systems and processes in the Model for Improvement.

What role I can play as a medical student if I were to observe or participate in an

$\begin{array}{ll}8.19(1.64) & P<0.001 \\ 7.87(1.48) & P<0.001 \\ 7.23(1.76) & P<0.001\end{array}$

adverse event, error, or systems failure.

$5.28(1.97)$

$P<0.001$

B. Skills

Question: Please rate your level of confidence in performing the following tasks

(1 low; 10 high)

I am able to identify an adverse event, error, or systems failure in my clinical work.

I am able to use the 5 Whys Tool to understand the contributing factors

underlying an adverse event, error, or systems failure.

I am able to propose a possible intervention to address an adverse event, error,

or systems failure.

I am able to reflect on possible unintended consequences of interventions

implemented to address an adverse event, error, or systems failure.

$\begin{array}{lll}6.51(1.97) & 8.32(1.60) & P=0.001 \\ 3.72(2.48) & 8.10(1.42) & P<0.001 \\ 5.63(1.99) & 7.94(1.50) & P<0.001 \\ 6.26(1.79) & 8.19(1.47) & P<0.001\end{array}$

Table 2 Summary analysis of students' free-text responses for elements to keep and elements to change in future sessions (with sample illustrative quotations)

\begin{tabular}{ll}
\hline \hline Elements to keep & Suggestions for improvement \\
\hline Facilitator with background in QI & Less prep work, clearer instructions \\
"Facilitator with knowledge of the subject matter" & "Less preparatory work - we could have gone over it as a group, and we would \\
"Great facilitator involvement; pointing out why things may have & $\begin{array}{l}\text { have all been on the same page going into the session" } \\
\text { happened and possible interventions with their pitfalls" }\end{array}$ \\
\end{tabular}

Case-based discussion of real scenarios

"Case discussion based on real scenarios students have experienced"

"Good to hear about the process in a real example"

Structured, guided reflection (e.g., 5 Whys Worksheet)

"I liked the 5 Why's sheet. I found it helpful to frame my reflection"

Time with fellow students

"Loved chances to talk with classmates and the faculty about issues we have seen and brainstorm solutions"

"Sharing our experience with our classmates and discussing the possible ways to address issues that have come up in the hospital"

Processing time between sessions (focusing first session on introduction, second session on debriefing)

"Thoughtfully designed with 2 sessions one month apart"

Identifying the role medical students can play

"Exploration of the role that medical students might play in patient safety/quality improvement"

Schedule dedicated time to complete reflection assignment

"Let us write during the work day (protected time)"

"Would love the exercise to have protected time during the work day for us to think and write"

More time for the session discussions

"Would like to get through more example cases. I really wanted to discuss my case but there wasn't time"

Provide information about current QI/safety initiatives at the local institution "More discussion of current patient safety/QI initiatives at [the hospital] with accompanying data would be helpful and illuminating"

"Hear more about [the hospital's] changes/interventions going on, real-world examples"

Feed forward student cases into the formal QI infrastructure at the institution; provide feedback on actions taken

"Real, detailed consideration of the problems we identified, and solutions proposed by Health and Safety, with feedback on any changes that took place as a result of this work"

"It would be good to have the suggestions be seen as taken seriously in some manner. For example, if we were told at the start that a panel of people from health and safety will consider our proposals and we will be contacted with the results and be informed of any changes that were made due to our ideas"

Sign-posting earlier in the year (introduce basic concepts early so students are on the lookout for cases ahead of this module)

" 1 . Put [the sessions] on the calendar next year so that future students know when to do the systems practice reflection and there is no confusion; 2 . Introduce the idea of patient quality and safety earlier (include a brief blurb during orientation?) so we are on the lookout for cases throughout the [core clinical year]"

"Make it clear that the session will happen later in the year so that students do not do this reflection early/without the context of the first quality \& safety session"

Demonstrate filing a safety report and discuss what happens next

"Having a faculty member who knew the official process and could tell us about it and what happens when a report is filed was helpful" 
implemented and may lead to a statistically significant increase in students' self-rated knowledge and skills. We designed this module to address the Association of American Medical Colleges' Entrustable Professional Activities goal of preparing students to "Identify systems failures and contribute to a culture of safety and improvement,"3 and feel confident it reached its objectives. Importantly, students felt better able to identify the role they can play if they observed or participated in an adverse event. ${ }^{4}$

The module design integrated principles of adult learning and Kolb's experiential learning cycle ${ }^{5}$ to support students to learn from their own experiences, actively reflect, and integrate new knowledge and skills into practice. We emphasized active, real-time, participatory learning, which student evaluations corroborated as being effective. We are exploring opportunities for connecting students' observations into the formal QI and patient safety infrastructure.

Limitations include a small sample size in a single academic institution. Response bias among students interested in QI is likely minimal given the mandatory nature of the session.

Medical students are positioned to identify, document, and explore opportunities to improve patient safety and quality. ${ }^{6}$ This session introduces students to concepts in QI and patient safety early in their training and prepares them to contribute to a culture of safety. Future research should evaluate the module's longitudinal impact on students' involvement in QI and safety throughout their training and career.

Acknowledgments: The authors wish to acknowledge Anjala V. Tess, MD, and Holly Gooding, MD, MSc, for their review of the session materials. Thank you to all of the Developing Physician faculty who supported this session: Kathryn Britton, MD, Joaquim M. Havens, $M D$, John Roseman, MD, Kristen Schaefer, MD, and Erik K. Alexander, MD, all of Brigham \& Women's Hospital. We also wish to specifically recognize and thank the Brigham \& Women's Hospital faculty members with quality improvement and patient safety expertise who facilitated the pilot sessions: Sonali Desai, MD, David Kroll, $M D$, and Peter Najjar, MD.

Corresponding Author: Galina Gheihman, MD; Department of Medicine Brigham and Women's Hospital, 75 Francis St., Boston, MA 02115, USA (e-mail: GGheihman@partners.org).

\section{Compliance with Ethical Standards:}

Conflict of Interest: The authors report no financial or other conflicts of interest.

Ethics Approval: Ethical approval was obtained from the Harvard Medical School Academy. The study was deemed quality improvement and waived from formal Institutional Review Board review.

\section{REFERENCES}

1. Lane-Fall MB, Davis JJ, Clapp JT, Myers JS, Riesenberg LA. What Every Graduating Resident Needs to Know About Quality Improvement and Patient Safety: A Content Analysis of 26 Sets of ACGME Milestones. Acad Medicine. 2018 Jun;93(6):904-910.

2. Wong BM, Etchells EE, Kuper A, Levinson W, Shojania KG. Teaching quality improvement and patient safety to trainees: a systematic review. Acad Med. 2010 Sep;85(9):1425-39.

3. Englander R, Flynn T, Call S, et al. Core entrustable professional activities for entering residency: Curriculum developers guide. Washington DC: Association of American Medical Colleges MedEdPORTAL iCollaborative; 2014. [Accessed July 24, 2019]. Resource ID 887. Available at: https:// www.aamc.org/system/files/c/2/482212-epa13toolkit.pdf. Accessed 15 March 2020.

4. Ryder HF, Huntington JT, West A, Ogrinc G. What Do I Do When Something Goes Wrong? Teaching Medical Students to Identify, Understand, and Engage in Reporting Medical Errors. Acad Med. 2019 Jul 16.

5. Kolb DA, Fry RE. (1974). Toward an applied theory of experiential learning. Cambridge, Mass: M.I.T. Alfred P. Sloan School of Management.

6. Gonzalo JD, Dekhtyar M, Hawkins RE, Wolpaw DR. How Can Medical Students Add Value? Identifying Roles, Barriers, and Strategies to Advance the Value of Undergraduate Medical Education to Patient Care and the Health System. Acad Med. 2017 Sep;92(9):1294-1301.

Publisher's Note: Springer Nature remains neutral with regard to jurisdictional claims in published maps and institutional affiliations. 